\title{
Collisional Activation of Ions in RF Ion Traps and Ion Guides: The Effective Ion Temperature Treatment
}

\author{
Aleksey V. Tolmachev, Andrey N. Vilkov, Bogdan Bogdanov, \\ Ljiljana Pǎsa-Tolić, Christophe D. Masselon, and Richard D. Smith \\ Pacific Northwest National Laboratory, Richland, Washington, USA
}

\begin{abstract}
Ion transfer and storage using inhomogeneous radio frequency (RF) electric fields in combination with gas-assisted ion cooling and focusing constitutes one of the basic techniques in mass spectrometry today. The RF motion of ions in the bath gas environment involves a large number of ion-neutral collisions that leads to the internal activation of ions and their effective "heating" (when a thermal distribution of internal energies results). The degree of ion activation required in various applications may range from a minimum level (e.g., slightly raising the average internal energy) to an intense level resulting in ion fragmentation. Several research groups proposed using the effective temperature as a measure of ion activation under conditions of multiple ion-neutral collisions. Here we present approximate relationships for the effective ion temperature relevant to typical operation modes of RF multipole devices. We show that RF ion activation results in near-thermal energies for ions occupying an equilibrium position at the center of an RF trap, whereas increased ion activation can be produced by shifting ions off-center, e.g., by means of an external DC electric field. The ion dissociation in the linear quadrupole ion trap using the dipolar DC ion activation has been observed experimentally and interpreted in terms of the effective ion temperature. (J Am Soc Mass Spectrom 2004, 15, 1616-1628) (C 2004 American Society for Mass Spectrometry
\end{abstract}

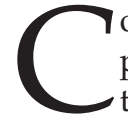
ollisional activation of ions takes place in all practical mass spectrometry measurements, either as a side effect of a residual gas pressure, or for the specific purpose of collisional ion cooling, focusing or collision induced dissociation. Modeling of the collisionally induced ion activation process in mass spectrometry has been the focus of extensive study [1-5]. Of particular importance is the collisional activation of ions in radio frequency (RF) ion traps and guides, where a substantial bath gas pressure and long residence times result in large numbers of the ionneutral collisions [6]. Several research groups have proposed using the effective temperature as a measure of ion activation under conditions of multiple ionneutral collisions [7-12]. As such, the effective temperature concept proved to be very useful in the interpretation of ion activation data obtained for various mass spectrometry experiments, in particular in quadrupole ion trap experiments $[8,9,13-15]$. Generally, the internal energy of ions produced at high pressures (e.g., by the electrospray ionization process) can be characterized in terms of effective ion temperature [16-20]. The

Published online September 22, 2004

Address reprint requests to Dr. R. D. Smith, Biological Systems Analysis and Mass Spectrometry, Pacific Northwest National Laboratory, 3335 Q Avenue (K8-98), P.O. Box 999, Richland, WA 99352, USA. E-mail: rds@pnl.gov effective temperature $T_{\text {eff }}$ of an ion, moving in a bath gas under the influence of electric fields, can be expressed through the ion drift velocity $V_{\text {drift }}$ as follows: $T_{\text {eff }}=T+$ $C_{T} V_{\text {drift }}^{2}$, where $T$ is the gas temperature, and $C_{T}$ is a model dependent proportionality coefficient $[9,12,21]$. This approximation is applicable to conditions when the force acting on an ion is constant during a time interval longer than the ion velocity relaxation time $\tau$ (i.e., the drift motion approximation) [22]. In the lower pressure regions of mass spectrometers, however, the conditions of drift motion are rarely encountered. For example, in an RF ion trap operating at a bath gas pressure of $\sim 1$ mTorr, the velocity relaxation time $\tau$ is on the order of $\sim 1 \mathrm{~ms}$, which is much longer than the characteristic time of a typical RF period, $\sim 1 \mu \mathrm{s}$. It follows that the collisional activation of ions due to fast RF motion in an RF ion trap is different than that of drift motion.

The aim of the present study is to further develop the effective temperature concept in the general case where the drift motion of ions is not necessarily established, e.g., for ions confined in RF ion traps. We consider the kinetics of collisional heating of ions using the characteristic ion heating time. It was previously suggested that this characteristic time is of the same order of magnitude as the velocity relaxation time $\tau$ [10]. Here, we present a more accurate estimation of the characteristic ion heating time based on recent findings for the 
thermal energy in polyatomic biomolecules, including peptide ions [23]. As a result, we obtain a simple relationship for the ion effective temperature as a function of ion kinetic energy, averaged over the ion heating time interval. We proceed with developing the relationship for the effective temperature as a function of local effective potential $V^{*}(r)$, a quantity that can be easily calculated for any given operating mode of an RF ion trap or ion guide $[6,24]$. Conditions for collision induced ion dissociation in a linear RF quadrupole ion trap, using an auxiliary dipolar DC potential, were evaluated theoretically and realized experimentally. Finally, we show the results of experiments testing the theoretical estimates.

\section{Theory}

The Effective Ion Temperature, Estimated Using the Thermal Analogy

The internal ion activation resulting from multiple ion-neutral collisions can be estimated using the thermal bath analogy. For this estimation, let us consider a case of a mono-atomic bath gas, where the energy exchange in each ion-neutral collision is defined by the ion internal state and the center-of-mass (COM) kinetic energy, and is independent of the origin of the kinetic energy. Ions moving in a bath gas environment and experiencing an acceleration from RF and DC fields will eventually acquire the same internal activation as the same ions under zero acceleration force, brought to thermal equilibrium with the bath gas heated up to a certain temperature $T_{\text {eff }}$ (see Appendix):

$$
k T_{\text {eff }}=\frac{2}{3}\left\langle K_{\mathrm{COM}}\right\rangle
$$

Here, $k$ is the Boltzmann constant and $\left\langle K_{\mathrm{COM}}\right\rangle$ is COM kinetic energy of the ion-neutral collision, averaged over a statistically large number of collisions:

$$
\begin{aligned}
& \left\langle K_{\text {СOM }}\right\rangle=\frac{m_{r}\left\langle v_{r e l}^{2}\right\rangle}{2}=\frac{m_{r}\left\langle v_{i}^{2}\right\rangle}{2}+\frac{m_{r}\left\langle v_{g}^{2}\right\rangle}{2} \\
& m_{r}=\frac{m m_{g}}{m+m_{g}}
\end{aligned}
$$

$\nu_{\text {rel }}$ is the relative ion-neutral velocity, $\nu_{\mathrm{i}}$ and $\nu_{\mathrm{g}}$ are velocities of the ion and gas molecules, respectively. The reduced mass $m_{r}$ is calculated from the ion mass $m$ and the gas molecular mass $m_{g}$. Eq 2 was derived in the same way as eq a5 in the appendix. Here the bath gas velocity conforms to a thermal velocity distribution, whereas the ion velocity has a generally undefined distribution. However the derivation used is based on general averaging rules and is valid for any form of the ion velocity distribution, not just the case of a thermal distribution.

The mean squared gas molecular velocity can be expressed via the gas temperature $T$ according to the classical relationship:

$$
\frac{m_{g}\left\langle v_{g}^{2}\right\rangle}{2}=\frac{3}{2} k T
$$

Substituting eqs 1 and 4 into eq 2 we obtain:

$$
T_{e f f}=\frac{m_{r}}{m_{g}} T+\frac{m_{r}\left\langle v_{i}^{2}\right\rangle}{3 k}
$$

which establishes the relationship between the internal activation of an ion and its mean squared velocity $\left\langle v_{i}^{2}\right\rangle$, a quantity well defined for any particular RF-DC collisional ion guide or trap. Ion trajectory calculations can be used to obtain $\left\langle v_{i}^{2}\right\rangle$ and then to estimate the resulting ion internal temperature $T_{\text {eff }}$ using eq 5 . The approximation does not require any assumption on the elasticity of collisions or details regarding the internal energy transfer mechanism. Moreover, neither ion-neutral cross section nor the bath gas pressure were involved in the derivation. The ultimate ion activation is defined only by the mean COM energy, and thus can be estimated using the thermal analogy.

One may argue that in the purely thermal case, the collision energy follows the thermal (i.e., Boltzmann) distribution, which is not the case for an ensemble of ions experiencing acceleration. However, the result of multiple step ion activation may be considered as a random walk process. The result of such a random walk for statistically large number of steps does not depend on the distribution of each step and is fully defined by the mean squared step length [25], in agreement with eq 5 . It can be shown that, under certain assumptions, the random walk ion excitation may result in the Boltzmann distribution of the internal ion energy, with the energy spread corresponding to the temperature (eq 5). We leave this consideration for a separate report.

The effective ion temperature relationship (eq 5) can be re-written in terms of the average laboratory frame (LAB) ion kinetic energy $\left\langle K_{i}\right\rangle$ as follows:

$$
T_{e f f}=\frac{m_{r}}{m_{g}} T+\frac{m_{r}}{m} \frac{2\left\langle K_{i}\right\rangle}{3 k}
$$

If the ion mass is large compared to the neutral mass, $m>>m_{g}$ then eq 6 can be simplified to:

$$
\begin{aligned}
& T_{\text {eff }} \approx T+\Delta T_{K} \\
& k \Delta T_{K}=\frac{2 m_{g}}{3 m}\left\langle K_{i}\right\rangle
\end{aligned}
$$


For example, for ions $m=1000$, the neutral mass $m_{g}=$ 40 (i.e., Ar bath gas) and the ion energy $\left\langle\mathrm{K}_{\mathrm{i}}\right\rangle=1 \mathrm{eV}$ we obtain:

$$
\Delta T_{K}=0.0267 \mathrm{eV} / \mathrm{k}=309 \mathrm{~K}
$$

Assuming that the bath gas is at room temperature, we arrive at $T_{\text {eff }} \approx 600 \mathrm{~K}$. Thus the ion kinetic energy sustained at $\sim 1 \mathrm{eV}$ results in the internal ion activation that corresponds to roughly double the room temperature.

\section{The Ion Activation Kinetics}

The effective ion temperature estimated above in eqs $5-8$ can be considered as a steady state temperature that is established as a result of a large number of collisions, with a sustained level of ion kinetic energy $\left\langle K_{i}\right\rangle$. The characteristic number of collisions needed to reach the steady state ion temperature can be obtained using the following estimation for ion-neutral energy exchange under the inelastic collision model.

The thermal energy in large polyatomic molecules shows a narrow distribution with energies centered on the mean thermal energy $E_{i}$, according to calculations for biomolecules, including peptides [23]. Consider an ensemble of ions having the thermal energy $E_{i}$ that evolves as a result of continuous collisional activation. The internal ion energy increment $\Delta E_{i}$ due to a single collision can be estimated as a difference of the initial energy $K_{C O M}$ and the COM kinetic energy of both projectiles after collision, $K^{\prime}{ }_{\mathrm{COM}}$ :

$$
\begin{aligned}
& K_{\mathrm{COM}} \approx C_{g} T_{e f f} \\
& K_{\mathrm{COM}}^{\prime} \approx C_{g} T_{i} \\
& \Delta E_{i} \approx C_{i} \Delta T_{i} \approx K_{C O M}-K_{C O M}^{\prime}
\end{aligned}
$$

Here $T_{i}$ is the time-dependent (non-stationary) ion temperature, $\Delta T_{i}$ is the mean ion temperature change in a single collision, and $C_{g}=3 \mathrm{k} / 2$ is the monoatomic molecular heat capacity. The energy and temperature values in eqs 10-12 should be considered as mean values, corresponding to averaging over the whole ensemble of ions. Eq 12 introduces the differential molecular heat capacity of an ion $C_{i}$. Eqs 10-12 correspond to the long-lived collision complex model, stating that the collision complex lifetime is sufficiently long for energy randomization [3]. The model gives the lower estimate for the characteristic ion heating time.

The number of collisions $\Delta n_{c}$ over a time interval $\Delta t$ is defined by the collision frequency $\nu_{c}$ as $\Delta n_{c}=\Delta t \nu_{c}$. We can thus estimate the time derivative of the ion temperature as follows:

$$
\begin{aligned}
& \frac{d T_{i}}{d t} \approx \frac{T_{e f f}-T_{i}}{\tau_{h}} \\
& \tau_{h}=\frac{C_{i}}{C_{g}} \frac{1}{v_{c}} \\
& v_{c}=n \sigma v_{r}
\end{aligned}
$$

where $n$ is the bath gas number density, $\sigma$ is the ion-neutral collision cross section and $\nu_{\mathrm{r}}$ is the relative ion-neutral velocity. Eqs 13-14 introduce the characteristic ion heating time $t_{h}$. The internal heat capacity of the ion is directly proportional to the size of the molecule for any particular class of compounds. The mean thermal energy of a molecule can be expressed as $E_{i}=$ $c(T, \nu) s k T$, where $s$ is the number of internal degrees of freedom [1, 23]. For peptide ions considered in [23] the $s$ number can be estimated using the ion molecular mass as $s \approx 0.4 \mathrm{~m}$. The $c(T, \nu)$ function between 300 and $500 \mathrm{~K}$ was assumed to be on the order of 0.2 [1]. For some organic compounds, including peptide ions, the function $c(T, \nu)$ can be approximated by the following quadratic function of temperature: $c(T, \nu) \approx c_{\text {peptide }}(T)=$ $c_{1} T-c_{2} T^{2}$, with coefficients $c_{1}=0.000561 \mathrm{~K}^{-1}$ and $c_{2}=$ $1.24 \cdot 10^{-7} K^{-2}$ [23]. The differential ion molecular heat capacity $C_{i}$ can then be estimated as the derivative of the ion internal energy with temperature: $C_{i}(T) \approx s k$ $\left(2 c_{1} T-3 c_{2} T^{2}\right)$. Substituting this relationship into eq 14 and using the approximation $s \approx 0.4 \mathrm{~m}$, we can obtain the characteristic ion heating time $\tau_{h}$ as a function of ion temperature and mass:

$$
\tau_{h}\left(T_{i}\right)=0.27 m v_{\mathrm{c}}^{-1}\left(2 c_{1} T_{i}-3 c_{2} T_{i}^{2}\right)
$$

For an order-of-magnitude estimation we can disregard the temperature dependence and replace the polynomial in eq 16 with a certain characteristic mass value $m_{0}$. For ion temperatures around $600 \mathrm{~K}$ we obtain the following estimation:

$$
\tau_{h}=\frac{m}{m_{0}} v_{c}^{-1} ; m_{0} \approx 7 u
$$

This characteristic time is of the same order of magnitude as the collisional relaxation time $\tau \approx$ $\left(\mathrm{m} / \mathrm{m}_{\mathrm{g}}\right) \nu_{c}^{-1}$ [22]. Consider the case of peptide ions and a bath gas with $m_{g}=28 \mathrm{u}$ at a pressure of $1 \mathrm{mTorr}$. Let us assume that $\sim 1000 \mathrm{u}$ ions have a collision cross section $s \approx 200 \AA^{2}$ and a relative velocity $\nu_{\mathrm{r}} \approx 500$ $\mathrm{m} / \mathrm{s}$, corresponding to the bath gas thermal molecular velocity. These values result in the following estimates for the collision frequency and the characteristic ion activation time:

$$
\nu_{\mathrm{c}} \approx 33000 \mathrm{~s}^{-1}
$$




$$
\tau_{h} \approx 0.004 s
$$

Integration of eq 13 using the temperature dependent $\tau_{h}\left(T_{i}\right)$ produces the kinetics for establishing the steady state ion temperature. For a simple estimation one can use the temperature-independent $\tau_{h}$ (eq 16a), which results in the following exponential kinetics:

$$
\Delta T_{\text {eff }}(t)=T_{\text {eff }}-T_{i}(t)=\Delta T_{\text {eff }}(t=0) \exp \left(-t / \tau_{h}\right)
$$

The time to reach the steady state temperature can be roughly estimated as $\tau_{s s} \sim 3 \tau_{h}$, which corresponds to $\exp \left(-t_{s S} / \tau_{h}\right) \approx 0.05$. In terms of number of collisions, it takes $3 m / m_{0} \approx 400$ collisions to reach the steady state ion temperature. This number is proportional to the ion mass within any particular class of compounds.

We are assuming that the thermal decomposition rate is negligibly small and does not affect the ion temperature. For polyatomic ions with $m>>m_{0}$ this condition realizes the high-pressure limit of the unimolecular decomposition reaction, or the rapid energy exchange (REX) limit. The REX limit is attained when the thermal dissociation constant is small compared to the inverse heating time.

The steady state ion temperature can be established provided the mean ion kinetic energy $\left\langle K_{i}\right\rangle$ is sustained during a sufficiently long time interval $t \geqslant t_{s s}$. Now we can define more exactly how the mean value $\left\langle K_{i}\right\rangle$ should be calculated in order to estimate the effective temperature using eqs 6-8. All fast variations of the instantaneous kinetic energy should be averaged over a time interval $\sim \tau_{h}$. These fast variations may include the ion acceleration between collisions, kinetic energy changes due to collisions, and fast kinetic energy variations due to the RF field acting on ions. The kinetic energy variations with a characteristic time much larger than $\tau_{h}$ should be considered as slow variations, and the corresponding effective ion temperature (eq 6) should be considered as a quasi-stationary, time-dependent quantity.

\section{The Effective Temperature Under the Effective Potential Approximation}

The relationships obtained above can be used to characterize the ion activation in collisional RF ion guides and traps. The ion motion in RF devices is modulated by RF oscillations. The ion kinetic energy $K_{i}$ can be divided into two approximate parts: the secular motion energy $K_{s}$ and the RF energy $K_{R F}$. An exact mathematical solution for the ion motion in quadrupolar RF fields gives an exact frequency spectrum describing the fast ion motion [26]. However, such a general solution does not exist for the case of RF multipoles. Fortunately, when the adiabaticity parameter is small, the ion motion can be well represented by oscillations with RF frequency $[6,24]$. This approximation has been used in the original derivation of the effective potential rela- tionship [24]. The term "micro-motion" is often used for the fast component of ion motion [6]. The exact frequency spectrum of the micro-motion is not essential for the present purposes. Important for ion activation, however, is the ion kinetic energy $K_{i}=K_{s}+K_{R F}$. Thus we exploit the fact that the micro-motion kinetic energy $K_{R F}$ is equal to the local effective potential value [6, 24].

The bath gas pressure needs to be sufficiently low so that the vacuum relationship for the RF ion velocity still holds. The pressure range can be estimated via the RF angular frequency $\omega$ and the collisional relaxation time $\tau$, using the inequality $\omega \tau>>1$ [22]. For an RF frequency of $\sim 1 \mathrm{MHz}$, the pressure limit is $p \lesssim 1$ Torr. Typically, the characteristic ion heating time $\tau_{h}$ is much larger than the RF period. For example, $\tau_{h} \sim 4$ ms estimated in eq 18 corresponds to $\sim 4000$ periods for $1 \mathrm{MHz}$ RF. Thus the kinetic energy of the RF ion motion $K_{R F}$ contributes to the mean energy $\left\langle K_{i}\right\rangle$ as the fast-changing component.

The ion energy component $K_{R F}$ can be estimated using the effective potential approximation (also called pseudo potential) $[6,24]$. The effective potential at a radius vector position $r$ is calculated for a given local RF field intensity $E_{R F}(r)$ as follows:

$$
V^{*}(r)=\frac{z e E_{R F}^{2}(r)}{4 m \omega^{2}}
$$

Here, $z e$ is the ion charge. The effective potential (eq 20) is expressed in $\mathrm{eV}$ units. The effective potential approximation gives reasonable results when the adiabaticity parameter is small, $\eta<<1$, which implies that the RF motion amplitude is small compared to the characteristic dimension of the RF potential spatial variation [6]. The mean kinetic energy of the RF ion motion under these conditions can be expressed via the local effective potential value:

$$
K_{R F}=\frac{m\left\langle v_{R F}^{2}\right\rangle}{2}=z e V^{*}
$$

Here, $\nu_{\mathrm{RF}}$ is the instantaneous velocity of the RF oscillations of ions; $K_{R F}$ is averaged over one RF period. Under the effective potential approximation, $K_{R F}$ acts as a potential, and the ion energy $K_{i}$ can be transferred from the secular to the RF energy component and vice versa.

Consider a case where the secular motion energy is negligibly small, $K_{s}=0$, and the ion position is defined by the balance between the confining force produced by the effective potential and an external static electric field $E$. This field can be created by the space charge of ions stored in the RF trap or it can be due to an applied DC potential gradient. For this example, we can estimate the ion effective temperature by substituting the ion energy (eq 6) with the RF energy (eq 21) as follows: 


$$
T_{e f f}=\frac{m_{r}}{m_{g}} T+\frac{m_{r}}{m} \frac{2 z e V^{*}}{3 k}
$$

We are assuming that the ion is balanced at a certain position for a time interval sufficient to reach the steady state level of the internal activation, $t \geqslant t_{s s}$. Note that although the secular energy is zero, ions still experience the collisional activation due to fast RF oscillations. The degree of activation of an ion oscillating at a certain effective potential $V^{*}$ is the same as for the sustained linear ion motion with the corresponding kinetic energy $K_{i}=z e V^{*}$. We proceed with examples of the effective ion temperature estimation in RF guides and traps.

\section{Ions Stored in an RF-Only Multipole Ion Trap; Low Space Charge Limit}

Ions trapped in an RF-only effective potential well are generally assumed to reach thermal equilibrium with the bath gas. The ion velocity is damped exponentially with the characteristic collisional relaxation time $\tau$. In the low space charge limit, the ions occupy positions close to the ion trap center at the effective potential minimum. Under steady state conditions the ion velocities describe a Boltzmann distribution, and the effective potential, averaged over the whole ion ensemble, can be estimated as $\left\langle V^{*}\right\rangle=3 / 2 \mathrm{kT}$ [22]. Substituting this value into eq 22 we obtain the following estimate for the ion effective temperature:

$$
T_{\text {eff }}=T
$$

i.e., the RF potential does not contribute to the ion temperature, which is defined only by equilibrium with the ambient gas. This ideal case is realized when the adiabaticity parameter is small, $\eta<<1$, as required by the effective potential approximation. Practical RF ion traps may operate under non-adiabatic conditions. For example, for a linear RF-only quadrupole where the adiabaticity parameter is equal to the q-parameter [26], increased $q$-values can cause an ion activation above the thermal level.

\section{Multipole Storage Assisted Ion Activation}

The coulomb repulsion of stored ions results in ion cloud expansion. The ions are shifted to off-center positions with increased RF field intensities, resulting in an increased kinetic energy of the RF oscillations. The ion activation due to the space charge from accumulated ions can be estimated as follows.

The linear charge density of ions stored in the RF multipole $Q_{l}$ is defined by the effective potential at the outer radial boundary of the ion cloud, $r=r_{q}$, as follows [27]:

$$
Q_{l}=4 \pi \varepsilon_{0}(N-1) V^{*}\left(r_{q}\right)
$$

where $\varepsilon_{0}$ is the dielectric constant and $2 N$ is the number of multipole rods. The effective potential $V^{*}\left(r_{q}\right)$ can be calculated from eq 24 and substituted into eq 22 to produce the effective ion temperature as a function of $Q_{l}$. Thus the space charge induced internal ion activation is defined by a given amount of trapped ions. This relationship can be used to interpret multipole storage assisted dissociation (MSAD) experiments [28-30]. Also one can use the relationship to predict the upper limit of the stored ion charge that is required for sufficiently low ion internal activation, e.g., for storage of fragile ion species [27].

\section{Secular Oscillations of Ions in an Effective Potential Well}

Another case of practical importance occurs in RF ion guides and ion traps, including the Paul trap. We begin with a case when the total ion kinetic energy $\left\langle K_{i}\right\rangle$ is sustained at a constant level, which in principle can be achieved by means of auxiliary ion excitation that exactly compensates collisional dissipation of the ion kinetic energy. From the effective potential approximation:

$$
K_{i}=K_{s}+K_{R F}=K_{s}+z e V^{*}(x, y, z)
$$

Here $x, y, z$ are the current secular motion coordinates of an ion. The ion kinetic energy is exchanged between the secular energy $K_{s}$ and the energy of RF oscillations $K_{R F}$ so that $K_{i}$ is constant. Under such conditions the mean kinetic energy $\left\langle K_{i}\right\rangle$ is equal to an instantaneous value $K_{i}$, and one can obtain the steady-state ion effective temperature by substituting $K_{i}$ (eq 25) into eq 6 . The dependence on the space $x, y, z$ coordinates is cancelled because the total energy is assumed to be constant. Note that the rate of ion collisional activation under such conditions does not depend on the secular motion phase and corresponds to the total kinetic energy $K_{i}$ at any point of the secular motion trajectory, either having a low effective potential and a high secular energy, or vice versa.

In general, the total ion kinetic energy $\left\langle K_{i}\right\rangle$ is time dependent and can gradually decrease, as in the case of collisional ion cooling, or increase with time, as in the case of auxiliary resonant ion excitation. The ion effective temperature should be estimated for these cases by using the relationship for ion heating kinetics, eq 13. In the case of a slow changing total kinetic energy $K_{i}(t)$ the ion effective temperature can be estimated as a quasi-stationary quantity, established over time intervals $\geqslant \tau_{h}$.

\section{Dipolar DC Activation of Trapped Ions}

As considered above, the ions stored in the RF trap can be activated by shifting their equilibrium to positions with higher RF field intensities, which can be achieved by applying an auxiliary dipolar DC potential gradient 


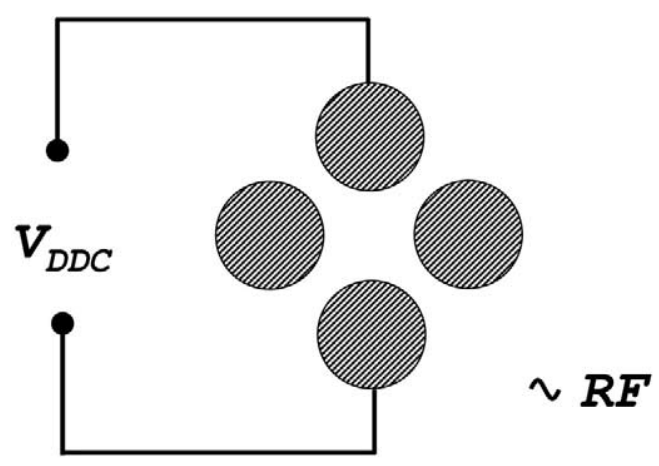

(a)

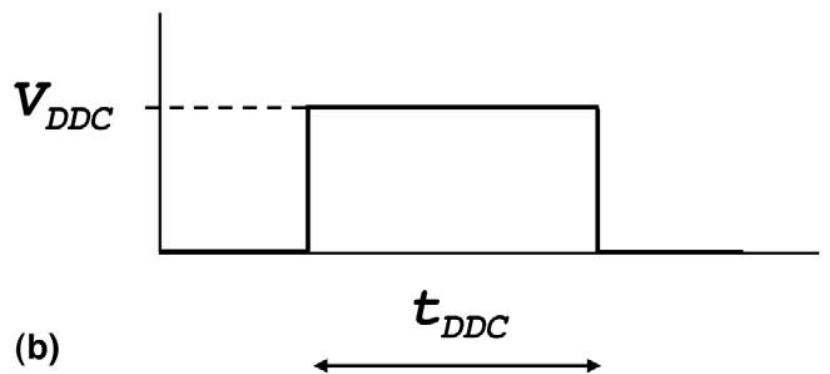

Figure 1. (a) Diagram of the RF quadrupole ion guide with the dipolar DC potential applied to a pair of opposite rods; (b) the dipolar DC pulse parameters include the amplitude $V_{D D C}$ and duration $t_{D D C}$.

across the ion trap. This approach can be realized in linear multipole RF ion traps, including quadrupole, hexapole, octopole and higher order traps, and also in the 3D quadrupole ion trap, i.e., the Paul trap. Nonresonant, low frequency dipolar AC activation of ions has been reported for the $3 \mathrm{D}$ quadrupole ion trap [31-33].

We now consider the linear RF-only quadrupole ion trap with an additional DC field applied orthogonally to the quadrupole axis (Figure 1a). The DC field is created by applying a DC offset $V_{D D C}$ to a pair of opposite quadrupole rods. We use the index DDC to distinguish the dipolar DC potential from the commonly used quadrupolar and trapping DC potentials. For our approximate consideration, we will assume that the DC field is spatially uniform and equal to:

$$
E_{D D C}=V_{D D C} / 2 r_{0}
$$

The linear quadrupole effective potential and effective focusing field are:

$$
\begin{aligned}
& V^{*}(r)=\frac{q V_{R F}}{4}\left(\frac{r}{r_{0}}\right)^{2} \\
& E^{*}(r)=-\frac{d V^{*}(r)}{d r}=-\frac{q V_{R F}}{2} \frac{r}{r_{0}^{2}}
\end{aligned}
$$

$$
q=\frac{4 z e V_{R F}}{m \omega^{2} r_{0}^{2}}
$$

Here, $V_{R F}$ equates to 0-peak RF amplitude and $r_{0}$ is the quadrupole inscribed radius. The radial position $r_{e}$ of the balance of the two fields, eqs 26 and 28, satisfies the following equation:

$$
E_{D D C}=-E^{*}\left(r_{e}\right)
$$

Using eqs 26-30, we obtain the effective potential at the point of equilibrium $r=r_{e}$ :

$$
V^{*}\left(r_{e}\right)=V_{D D C}^{2} / 4 q V_{R F}
$$

which can be substituted into eq 22 to obtain the steady state ion effective temperature. For a simple estimation, consider the case $m>>m_{g}$. Substituting $\left\langle K_{i}\right\rangle$ in eq 8 by $z e V^{*}\left(r_{e}\right)$ we obtain:

$$
k \Delta T_{K}=\frac{z e}{6 q} \frac{m_{g}}{m} \frac{V_{D D C}^{2}}{V_{R F}}
$$

This relationship allows one to define the effective ion temperature for any given RF quadrupole parameters. Substituting $q$ from eq 29 into eq 32, we obtain the following expression:

$$
k \Delta T_{K}=\frac{m_{g} \omega^{2} r_{0}^{2}}{24}\left(\frac{V_{D D C}}{V_{R F}}\right)^{2}
$$

This expression indicates the effective temperature is independent of ion mass $m$ and charge ze: all trapped ion species are activated to the same effective temperature (eq 33). The equilibrium position $r_{e}$ is $\mathrm{m} / \mathrm{z}$ dependent, as can be obtained from eqs 27 and 31:

$$
r_{e}=\frac{r_{0}}{q} \frac{V_{D D C}}{V_{R F}} \propto m / z
$$

The off-axis displacement $r_{e}$ is proportional to $m / z$. High $\mathrm{m} / \mathrm{z}$ ions with $r_{e} \geqslant r_{0}$ will strike a quadrupole rod and be lost. The corresponding high $\mathrm{m} / \mathrm{z}$ cut-off condition can be obtained from eq 34 by substituting $r_{e}<r_{0}$ :

$$
m / z<(m / z)_{\text {high }} \equiv q_{u} \frac{V_{R F}}{V_{D D C}}
$$

Here the coefficient $q_{u}$ is defined as the $q$ parameter (eq 29), calculated for $m / z=1 \mathrm{Th}$. This coefficient can also be used to provide a simple estimation of the low $\mathrm{m} / \mathrm{z}$ cut off in RF-only quadrupoles, as follows:

$$
(m / z)_{l o w}=1.1 q_{u}
$$


The coefficient $1.1 \approx(0.908)^{-1}$ results from the ion stability condition $q<0.908$ in RF-only quadrupoles [26]. The RF quadrupole with a dipolar DC potential acts as a low-pass mass filter, with a mass range given by eqs 35 and 36. Similar results have been reported for the 3D ion trap [33]. The side-effect of such a low-pass filter is the ion activation due to the DDC field.

The relationship in eq 35 should be considered as an approximation. More accurate values can be obtained by using direct simulations or an exact theory [34]. Such a consideration must take into account the realistic DDC pulse shape, since the trajectory of an ion that approaches equilibrium at $r=r_{e}$ is very sensitive to the DDC pulse rise time $t_{p}$. We have conducted simulations, using the model pulse front, corresponding to the integration RC circuit: $V(t)=V_{D D C}\left[1-\exp \left(-t / t_{p}\right)\right]$. Simulations have shown that the secular trajectory of an ion gradually approaches the equilibrium position (eq 34) for the following parameters: $t_{p} \geqslant 0.01 \mathrm{~ms}, \omega=$ $2 \pi \cdot 10^{6} \mathrm{~Hz}$. Short rise times, $t_{p} \lesssim 1 \mu \mathrm{s}$, result in increased secular ion energies with ion trajectories reaching high radial positions, $r>>r_{e}$. It follows that the controlled pulse rise time is needed in order to approach conditions of the minimum secular energy assumed above. Generally, the approximation (eq 35) for high $m / z$ limit can be used for $\omega t_{p}>>1$ and $q<\sim 0.5$.

The relationships presented above in eqs 32-35 can be used to find experimental conditions for the ion dissociation in an RF quadrupole using DDC ion activation. We can estimate the DDC potential required to obtain ion dissociation as follows. The effective ion temperature for the ion dissociation in a $\sim 10 \mathrm{~ms}$ time frame can be estimated from the Arrhenius parameters obtained for peptides from blackbody infrared radiative dissociation (BIRD) experiments [35-40]. For an initial estimate, we assume $T_{\text {eff }} \sim 700 \mathrm{~K}$, or in terms of eq $32, \Delta T_{K} \sim 400 \mathrm{~K}$. The parameters used for our experimental RF quadrupole setup are as follows: RF voltage $V_{R F}=150 \mathrm{~V}$ (0-peak), frequency $710 \mathrm{kHz}$, inscribed quadrupole radius $r_{0}=2.8$ $\mathrm{mm}$, bath gas $m_{g}=28$ (nitrogen), pressure $\sim 4 \mathrm{mTorr}$. The $q$-parameter (eq 29) estimated for $m / z=1000$ is $q=0.37$. From eq 32 we obtain $V_{D D C} \sim 20 \mathrm{~V}$. The equilibrium position, eq 34, is $r_{e} \sim 1 \mathrm{~mm}$. Thus a minor off-axis displacement should be sufficient for reaching ion dissociation offset. The ion $\mathrm{m} / \mathrm{z}$ range estimated from eqs 35 and 36 is 409 to 2780 Th. The duration of DDC ion activation, required to reach the steady state effective temperature, is $t_{D D C} \geqslant t_{s s} \sim 3 \mathrm{~ms}$, as can be estimated using eq 16a. The conditions of the DDC ion dissociation obtained, $V_{D D C} \sim$ $20 \mathrm{~V}$ and $t_{D D C} \sim 10 \mathrm{~ms}$, were tested experimentally, as considered in the following section.

\section{Experiment}

The conditions for DDC ion dissociation were realized in a linear RF quadrupole ion trap, Figure 1a. Experiments were carried out on our recently modified 7 tesla FTICR mass spectrometer [41, 42]. The instrument incorporates an ESI ion source with an electrodynamic ion funnel, an
RF octopole for collisional cooling, and a set of RF quadrupoles for ion pre-selection, accumulation and transfer to the orthorhombic ICR cell. The quadrupole ion guide setup was modified to perform external accumulation and collisional induced dissociation in the first quadrupole, $\mathrm{Q}_{0}$. The quadrupole was operated at a pressure $\sim 4 \times 10^{-3}$ Torr of background air, and was driven by an in-housebuilt high-Q head controlled by a function generator and an RF amplifier, $300 V_{p-p}$ at $710 \mathrm{kHz}$. Fibrinopeptide A (Sigma Chemicals, St. Louis, MO), was dissolved in a water/methanol/acetic acid solution (49:49:2 vol\%) at a concentration $10^{-6} \mathrm{M}$. The solution was infused into the ESI source at a flow rate of $300 \mathrm{~nL} / \mathrm{min}$ using a syringe pump (Harvard, South Natick, MA). The ions were accumulated in $\mathrm{Q}_{0}$ during $3 \mathrm{~s}$ and cooled for $0.1 \mathrm{~s}$. Next, the dipolar DC potential $V_{D D C}$ was applied over a certain time interval $t_{D D C}$ (Figure 1b). A second cooling period of $0.1 \mathrm{~s}$ was set after $V_{D D C}$ was switched off. Ions were then ejected from $Q_{0}$ and transferred into the ICR cell for mass spectra acquisition.

For unperturbed conditions when $V_{D D C}=0 \mathrm{~V}$, only the parent fibrinopeptide A $2+$ peak at $m / z=768$ was observed. When $V_{D D C} \geqslant 20 \mathrm{~V}$, ion dissociation was observed (see Figure 2a and b). The degree of parent ion dissociation depended sharply on both the DDC voltage $V_{D D C}$ and the time interval $t_{D D C}$. In the case of $V_{D D C}$ $=34 \mathrm{~V}$ shown in Figure 2, the increase of $t_{D D C}$ from 0.8 $\mathrm{ms}$ (Figure 2a) to $0.9 \mathrm{~ms}$ (Figure $2 \mathrm{~b}$ ) resulted in a sharp decrease of the parent peak intensity.

The fragmentation pattern consisting of $y$ - and $b$-type fragment ions is characteristic of the soft fragmentation mode and is consistent with multiple collision low kinetic energy ion activation. Ion fragments having $\mathrm{m} / \mathrm{z}$ values both lower and higher than the precursor ion $\mathrm{m} / \mathrm{z}$ value were observed, proving their stable ion confinement under the DDC voltage conditions.

The parent ion intensity profiles versus the DDC voltage are shown in Figure 3. The dissociation offset voltage was higher for shorter time intervals $t_{D D C}$. For ion activation times $t_{D D C}>10 \mathrm{~ms}$, the dissociation offset voltage decreased just slightly compared to $V_{D D C}=20$ $\mathrm{V}$ obtained for $t_{D D C}=10 \mathrm{~ms}$, indicating that the steady state level of ion activation was reached over a $\sim 10 \mathrm{~ms}$ time frame.

The DDC voltage increase above the fragmentation offset resulted in reduction of the fragment peak intensities, which can be attributed to the dissociation of fragment ions. Figure 4 shows the fragment peak intensity versus $V_{D D C}$ for $t_{D D C}=2 \mathrm{~ms}$.

The fragmentation pattern changed depending on the values selected for $t_{D D C}$ and $V_{D D C}$. Figure 5 shows maximum $y$ - and $b$-fragment intensities observed for five different DDC activation times. Shorter activation times in Figure 5 correspond to higher DDC voltages, and correspondingly higher ion temperatures, as estimated in the next section (see Table 1). Thus the behaviors shown in Figure $5 \mathrm{a}$ and $\mathrm{b}$ can be attributed to the competition of different ion decomposition channels that have different activation energies. 


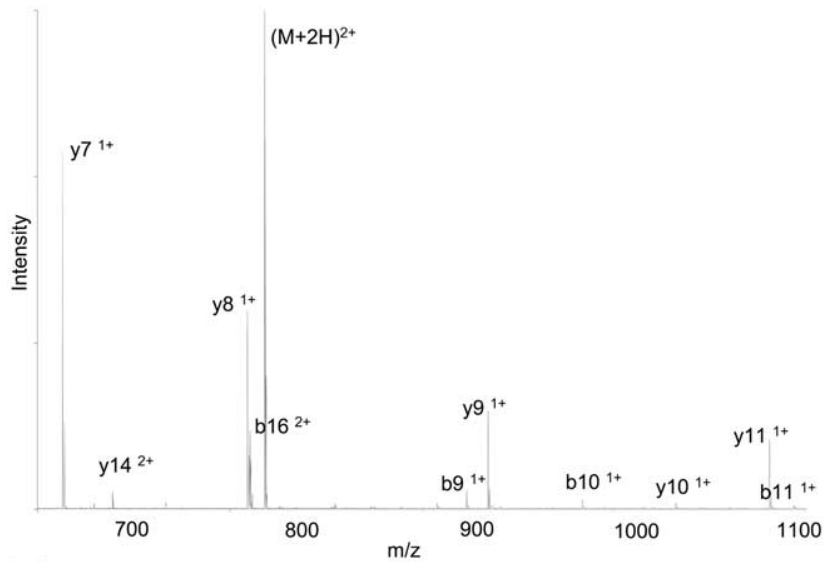

(a)

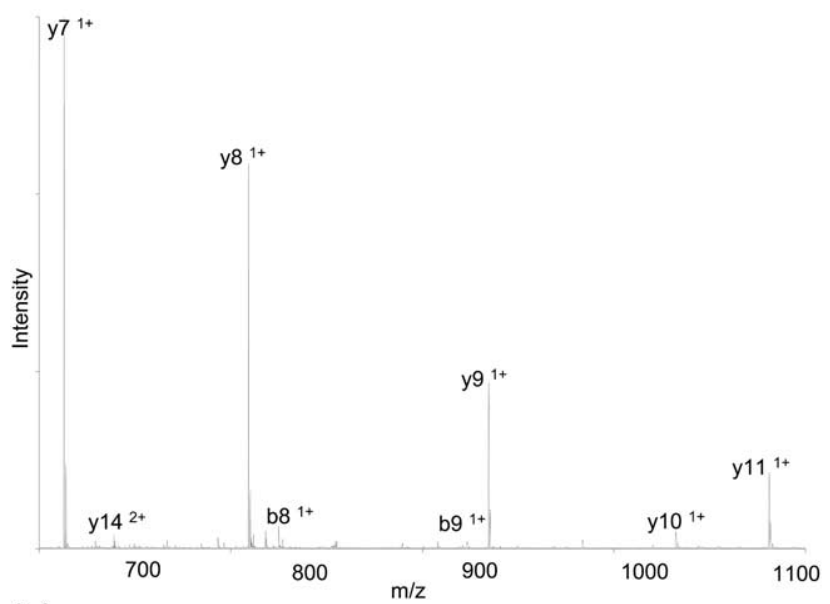

(b)

Figure 2. Ion dissociation spectra for fibrinopeptide $\mathrm{A}$ ions, obtained for $V_{D D C}=34 \mathrm{~V}$. The time interval of the dipolar DC ion activation is (a) $t_{D D C}=0.8 \mathrm{~ms},(\mathbf{b}) t_{D D C}=0.9 \mathrm{~ms}$.

\section{Discussion}

In this section we model the experimental ion dissociation curves (Figure 3) in order to further test theoretical predictions. A simple formalism described below establishes a connection between the degree of parent ion dissociation and the effective ion temperature, using the Arrhenius thermal decomposition parameters.

The ion dissociation conditions observed experimentally agree with the theoretical estimate, $V_{D D C} \approx 20 \mathrm{~V}$ for $t_{D D C}=10 \mathrm{~ms}$.

The higher potentials needed for shorter activation time intervals, Figure 3, can be attributed to two factors. First, the steady state effective temperature is not established for $t_{D D C} \lesssim 3 \mathrm{~ms}$, as was estimated theoretically. Second, shorter $t_{D D C}$ intervals require faster decomposition reaction rates, and thus higher effective temperatures. Parent ion dissociation curves in Figure 3 can be modeled using the unimolecular decomposition formalism. The number of ions experiencing decomposition in the time interval $d t$ is:

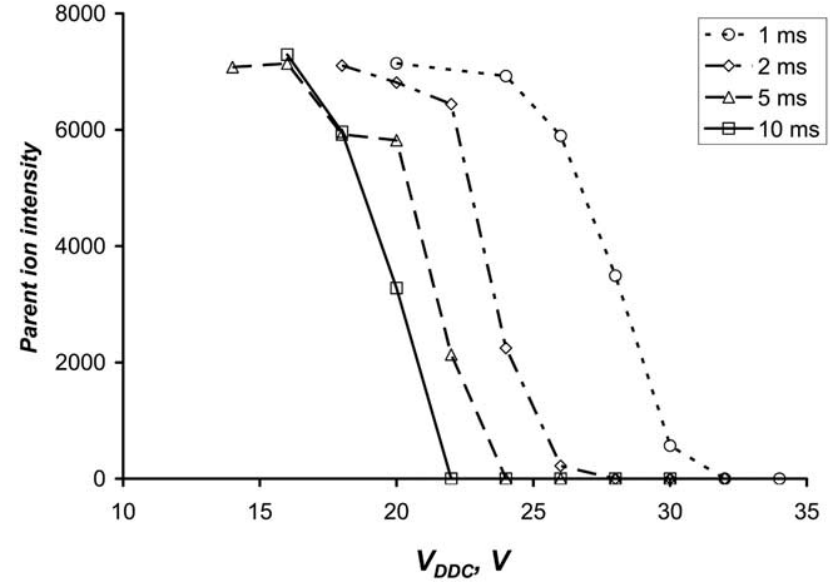

Figure 3. Parent ion intensities (fibrinopeptide A $2+, m / z$ 768.85) versus the dipolar DC voltage for dipolar DC pulse intervals $t_{D D C}$ $=1,2,5$, and $10 \mathrm{~ms}$.

$$
d n=n(t) k_{p} d t
$$

where $n(t)$ is the time dependent number of parent ions and $k_{p}$ is the unimolecular decomposition reaction rate constant for parent ions. We can take ion temperature variations into account using the temperature dependent reaction constant $k_{p}\left(T_{i}\right)$. Here $T_{i}$ is the timedependent (non-stationary) ion temperature, introduced in eqs 11 and 13. We assume that multiple channels of the parent ion dissociation can be approximated by a single set of Arrhenius parameters, the pre-exponential factor $A$ and activation energy $E_{a}$ :

$$
k_{p}\left(T_{i}\right)=A \exp \left(-E_{a} / k T_{i}\right)
$$

Integration of eq 37 yields the following kinetic expression for the parent ion abundance:

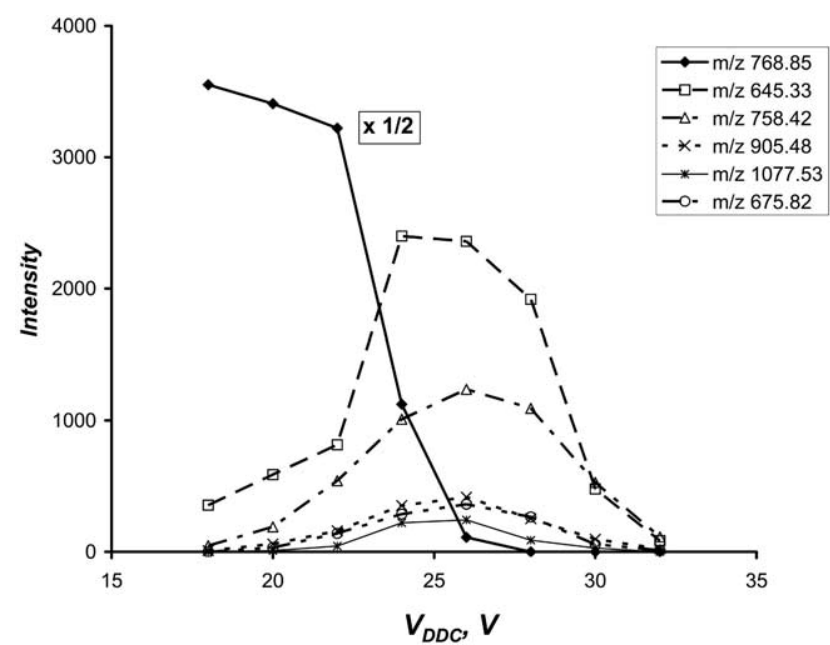

Figure 4. Fragment peak intensities versus $V_{D D C}$, for $t_{D D C}=2$ $\mathrm{ms}$. The parent peak intensity, fibrinopeptide A $2+, \mathrm{m} / \mathrm{z} 768.85$, is scaled by 0.5 . Fragments shown are: $\mathbf{y}_{7}{ }^{1+}(\mathrm{m} / \mathrm{z} 645.33), \mathrm{y}_{8}{ }^{1+}(\mathrm{m} / \mathrm{z}$ 758.42), $\mathrm{y}_{9}{ }^{1+}\left(m / z\right.$ 905.48), $\mathrm{y}_{11}{ }^{1+}\left(m / z\right.$ 1077.53), $\mathrm{y}_{14}{ }^{2+}(m / z$ 675.82). 


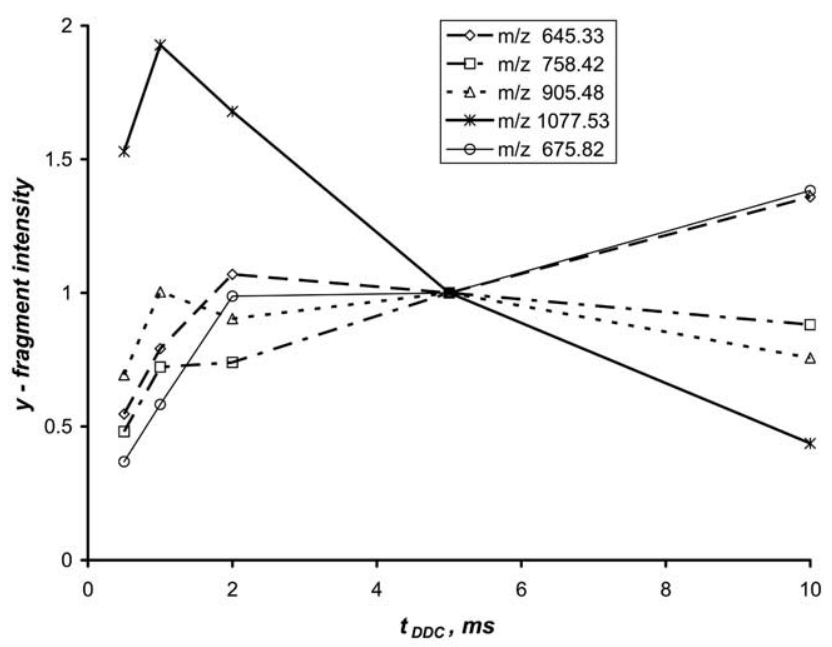

(a)

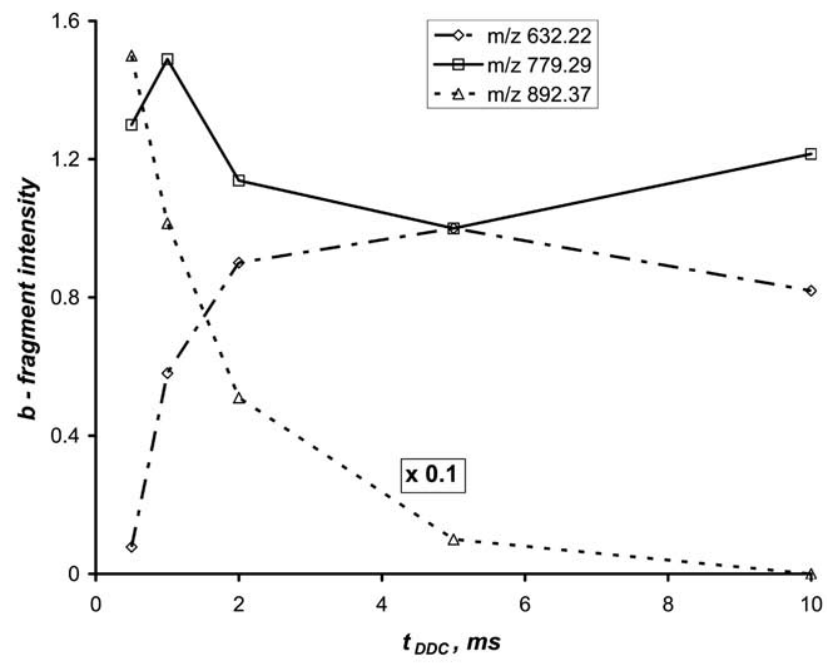

(b)

Figure 5. Fragment peak intensities obtained for different DDC pulse durations. The DDC voltage for each point corresponds to the maximum fragment intensity obtained for a given $t_{D D C}: V_{D D C}$ $=38,35,26,24,22 \mathrm{~V}$ for $t_{D D C}=0.5,1,2,5,10 \mathrm{~ms}$. Intensities are normalized by values for $t_{D D C}=5 \mathrm{~ms}$. (a) y-fragments: $\mathrm{y}_{7}{ }^{1+}(\mathrm{m} / \mathrm{z}$ 645.33), $\mathrm{y}_{8}{ }^{1+}(\mathrm{m} / \mathrm{z} 758.42), \mathrm{y}_{9}{ }^{1+}\left(\mathrm{m} / \mathrm{z}\right.$ 905.48), $\mathrm{y}_{11}{ }^{1+}(\mathrm{m} / \mathrm{z}$ 1077.53), $\mathrm{y}_{14}{ }^{2+}{ }^{(m / z}$ 675.82); (b) b-fragments: $\mathbf{b}_{7}{ }^{1+}\left(\mathrm{m} / \mathrm{z}\right.$ 632.22) $\mathbf{b}_{8}{ }^{1+}(\mathrm{m} / \mathrm{z}$ 779.29) (intensity is scaled by 0.1$), \mathbf{b}_{9}{ }^{1+}(\mathrm{m} / \mathrm{z}$ 892.37).

$$
n(t)=n_{0} \exp \left(-t_{r}\right)
$$

where $t_{r}$ the dimensionless reaction time:

$$
t_{r}=\int_{0}^{t} k_{p}\left(T_{i}\right) d t
$$

This integral can be calculated using the timedependent ion temperature given by eq 13 , with the temperature-dependent characteristic heating time, eq 16. The steady state ion temperature $T_{\text {eff }}$ in eq 13 is calculated as a function of the experimental parameters, eq 33.

The parent ion dissociation kinetics, eq 39, has been calculated by means of direct numerical integration from eqs 13 and 40. Figure 6 shows calculated relative parent ion abundance versus $V_{D D C}$ for different $t_{D D C}$ intervals. Unknown parameters $A, E_{a}$ and $\sigma$, were adjusted to fit the experimental data shown in Figure 3, and the following values were obtained:

$$
A=1 \cdot 10^{11}, \quad E_{a}=1.2 e V, \quad \sigma=400 \AA^{2}
$$

The ion-neutral collision cross section $\sigma$ corresponds to the experimental pressure of $4 \mathrm{mTorr}$. The uncertainty in the pressure measurements $(\sim 50 \%)$ contributes to the relative error of the cross section estimation. Additional uncertainty is due to the inelastic ionneutral collision approximation used in deriving the characteristic heating time, eq 16. The cross section value obtained for Fibrinopeptide A, molecular weight (MW) 1536, is consistent with an empiric rule for the ion cross section of the denatured biomolecular ions, $\mathrm{MW} / \sigma=5 \mathrm{Th} / \AA^{2}[43,44]$, which gives $\sigma \approx 1536 / 5 \approx$ $300 \AA^{2}$.

The activation energy and pre-exponent factor (eq 41) agree with the Arrhenius parameters obtained in BIRD experiments for peptide ions [45-47]. We conclude that the experimental data are consistent with our theoretical model.

Calculation of the extent of dissociation includes integration of the effective temperature using eqs 13 and 16. As a result, the time-dependent effective temperature $T_{i}(t)$ is obtained for each set of $V_{D D C}$ $t_{D D C}$ parameters. The function $T_{i}(t)$ reaches a steady state with the characteristic time $\tau_{h}$ and relaxes to room temperature after the end of the DDC pulse. Table 1 shows calculated effective ion temperatures and characteristic heating times. $V_{D D C}$ values correspond to $50 \%$ depletion of the parent ion abundance for each of the four listed $t_{D D C}$ values. $T_{i}$ values shown correspond to maximum ion effective temperatures achieved during ion activation (i.e., at the end of the DDC pulse) for corresponding $t_{D D C}$ and $V_{D D C}$ values. Steady-state effective ion temperatures $T_{\text {eff }}$ are calculated using eq 33 for each $V_{D D C}$ value, and characteristic heating times $\tau_{h}$ are calculated for each $T_{i}$ value using eq 16 and the collision cross section obtained from the fitting procedure.

Ion temperatures $T_{i}$ (Table 1) approach corresponding steady state temperatures $T_{\text {eff }}$ for suffi-

Table 1. Parameters, corresponding to $50 \%$ parent ion dissociation in Figure 6: Dipolar DC pulse duration $t_{D D C}$ and amplitude $V_{D D C}$, the effective ion temperature $T_{i}$, the steadystate ion temperature $T_{\text {eff }}$ and the characteristic ion heating time $t_{h}$ eq 16

\begin{tabular}{ccrrr}
\hline$\tau_{D D C}, m s$ & $V_{D D C^{\prime}} V$ & $T_{i,} K$ & $T_{\text {eff, }} K$ & $\tau_{h^{\prime}} m s$ \\
\hline \hline 1.0 & 28.00 & 804.1 & 1036.9 & 1.077 \\
2.0 & 23.75 & 771.3 & 826.8 & 1.049 \\
5.0 & 21.00 & 707.5 & 709.2 & 0.989 \\
10.0 & 20.00 & 670.0 & 670.0 & 0.952 \\
\hline
\end{tabular}




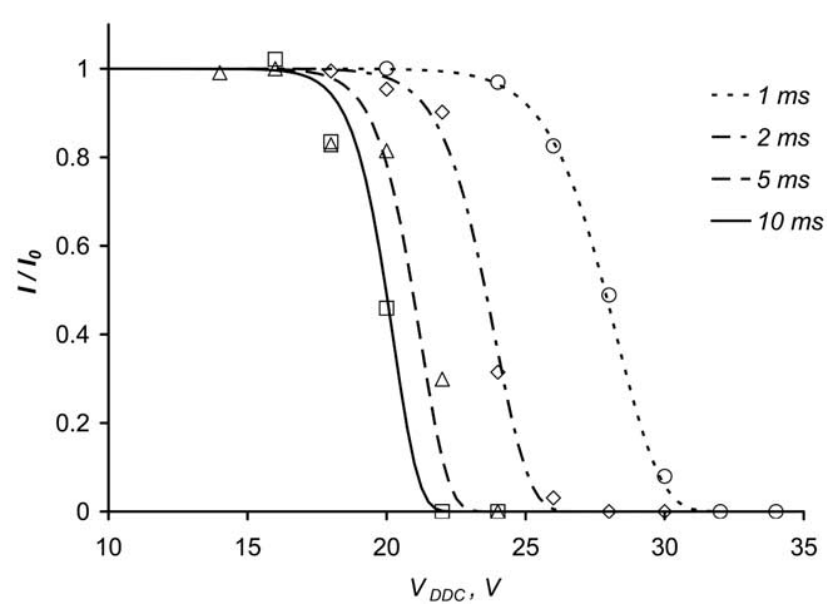

Figure 6. Relative parent ion intensities versus the dipolar DC voltage for different dipolar DC pulse intervals. Theoretical curves are fitted to experimental data from Figure 3, using the following parameters: pre-exponent $A=1 \times 10^{11}$, activation energy $E_{a}=1.2$ $\mathrm{eV}$ and the ion-neutral collision cross section $\sigma=400 \mathrm{~A}^{2}$.

ciently long DDC activation times, $t_{D D C}=5$ and 10 $\mathrm{ms}$, consistent with the theoretical estimation of the steady state regime, $t_{D D C}>3 \tau_{h}$. Interpretation of the dissociation curves, such as shown in Figure 3, can be greatly simplified for such conditions, where the ion fragmentation is mostly defined by the steady state temperature $T_{\text {eff, }}$, sustained over a predefined time interval $t_{D D C}$. The reaction rate is small compared to the ion activation rate, signifying that the REX limit is realized. These conditions are similar to classical thermo-chemical conditions, where the temperature of the system and the reaction time interval are well defined and the high-pressure limit for the reaction constant is realized. The unimolecular rate constants can then be obtained for each of the reaction channels observed. One possible approach is to use the initial reaction stage, where the abundance $I_{i}$ of the product ions of a type $i$ increases linearly with the reaction time. The reaction constant for the $i$-th channel can be determined as follows:

$$
k_{i}=\frac{I_{i}\left(t_{D D C}\right)}{I_{0} t_{D D C}}
$$

Here, $I_{0}$ is the initial abundance of the precursor ions. The reaction rate constant $k_{i}$ can be measured for a set of different effective ion temperatures in order to obtain corresponding Arrhenius parameters $A_{i}$ and $E_{a i}$ for the $i$-th fragment. Arrhenius plots were used previously to obtain pre-exponential factors and activation energies from the ion dissociation spectra in BIRD experiments [35-40]. Our method can provide higher ion temperatures; thus a larger number of fragmentation channels can be observed for any given precursor ion species. Faster dissociation times of $\sim 0.01 \mathrm{~s}$ will permit higher throughput studies of ion dissociation under controlled ion effective temperature.

\section{Conclusions}

We have shown that the effective ion temperature approach can be used to estimate the degree of ion activation in RF ion traps and multipole guides. The theoretical treatment shows the basis for a new experimental approach for the dissociation of ions trapped in RF ion traps, using the dipolar DC potential applied across the RF ion trap. The method is applicable to linear multipole RF ion traps, including quadrupole, hexapole, octopole, and higher order traps, and also to the 3D quadrupole ion trap, i.e., the Paul trap. A linear RF quadrupole ion trap was used in our experimental study. The effective temperature of ions was estimated as a function of the experimental parameters. The data obtained is consistent with corresponding values reported for BIRD experiments with peptide ions [45-47].

Unlike boundary activated ion dissociation (BAD) [13, 48-51], DDC ion dissociation allows the stable ion confinement of fragments, with $\mathrm{m} / \mathrm{z}$ values both higher and lower than the parent $\mathrm{m} / \mathrm{z}$ value, making the approach applicable for multiply charged ions, e.g., produced by an electrospray ion source. Another advantage is the ability to set a desired ion activation intensity that can be characterized in terms of the effective ion temperature. The method can be operated in the slow ion heating mode, where the ion activation takes a large number of ion-neutral collisions. This mode requires setting a combination of low DDC voltage and long DDC ion activation time.

The DDC ion dissociation proposed is different from the non-resonance dipolar ion activation in ion traps considered previously [31-33], where a low frequency AC dipolar potential results in repeated ion activation events. We propose using a single DDC pulse, the duration of which is adjusted for a desired number of ion-neutral collisions.

DDC ion activation can be of a great practical utility as an alternative ion dissociation approach that is less sensitive to ion space charge effects than the resonant CID approaches. A fine adjustment of the ion activation is enabled by means of two well-controlled parameters: the DDC potential and the DDC time interval. The time frame of $\sim 1 \mathrm{~ms}$ and the pressure of $\sim 1$ mTorr are consistent with existing ion guide setups used for ion cooling, external pre-selection and accumulation.

High effective temperatures, $\sim 1000 \mathrm{~K}$, achievable using DDC activation, enable short, $\sim 1 \mathrm{~ms}$, ion dissociation times. High ion temperatures result in dissociation of high energy bonds, and the number of fragments obtained should be similar to the number obtained using other CID approaches. It is possible to obtain Arrhenius activation energies for each of the fragmentation pathways. This ability can be used to further improve specificity of peptide and protein identification in mass spectrometrybased proteomics studies.

It will also be attractive to implement DDC dissociation in a $3 \mathrm{D}$ quadrupole ion trap. The effective ion 
temperature for DDC ion activation in an ion trap can be defined in a similar manner to a linear RF quadrupole. Bath gas conditions of $\sim 1$ mTorr He would allow one to attain the steady state ion temperature using DDC pulse intervals $t_{D D C} \geqslant 3 \tau_{h} \approx 6 \mathrm{~ms}$. Thus ion trap MS/MS measurements could be supplemented by a fast DDC-heating of ions up to a desired effective temperature, with possible determination of the Arrhenius parameters for dissociation channels observed.

\section{Acknowledgments}

The authors thank Drs. Julia Laskin, Eric Strittmatter, and Anil Shukla for helpful discussions, Dr. Seonghee Ahn for assistance in experimental measurements, and David Prior for electronics support. Portions of this research were supported by the NIH National Center for Research Resources (RR18522) and by the U.S. Department of Energy's Office of Biological and Environmental Research. The Pacific Northwest National Laboratory is operated by Battelle for the U.S. Department of Energy through contract DE-AC06-76RLO 1830.

\section{Appendix}

One can draw an analogy between ions experiencing multiple ion-neutral collisions and an ensemble of molecules immersed in a bath gas with a certain temperature $T$. The internal energy of the molecules reaches a level, corresponding to the bath gas temperature, after a time interval, sufficient for the thermal equilibrium to be established. On the other hand, the internal energy of ions can be estimated using the center of mass (COM) energy of ion-neutral collisions. Under the slow ion heating conditions the internal ion energy increases gradually and reaches the steady state level, defined by the COM collision energy. Thus, the relationship between the COM energy and temperature for the bath gas can be used to estimate the effective ion temperature, established as a result of sufficiently large number of ion-neutral collisions with a corresponding COM energy.

Consider a mixture of two gases in thermal equilibrium at temperature $T$. The mean kinetic energy of the molecules in the laboratory frame is as follows:

$$
\frac{m_{1}\left\langle v_{1}^{2}\right\rangle}{2}=\frac{m_{2}\left\langle v_{2}^{2}\right\rangle}{2}=\frac{3}{2} k T
$$

Here $m_{1}, m_{2}, \nu_{1}$, and $\nu_{2}$ are molecular masses and velocities of each of the two gases; angular brackets designate mean values.

The center of mass (COM) collision energy for a collision of two different molecules is as follows:

$$
\left\langle K_{C O M}\right\rangle=\frac{m_{r}\left\langle v_{r e l}^{2}\right\rangle}{2}
$$

$$
\begin{gathered}
m_{r}=\frac{m_{1} m_{2}}{m_{1}+m_{2}} \\
\overrightarrow{v_{r e f}}=\overrightarrow{v_{1}}-\overrightarrow{v_{2}}
\end{gathered}
$$

$m_{r}$ is the reduced mass and $\nu_{r e l}$ is the relative velocity of the two molecules. The mean value of the scalar product of two vectors is zero if at least one of the vectors has random orientation, thus taking mean value of the COM energy (eq a2) results in:

$$
\left\langle K_{\text {COM }}\right\rangle=\frac{m_{r}}{2}\left\langle v_{1}^{2}+v_{2}^{2}-2 \vec{v}_{1} \vec{v}_{2}\right\rangle=\frac{m_{r}\left\langle v_{1}^{2}\right\rangle}{2}+\frac{m_{r}\left\langle v_{2}^{2}\right\rangle}{2}
$$

Substituting eq a1 and eq a3 into eq a5 we arrive at the sought relation between the temperature and the COM kinetic energy:

$$
\left\langle K_{\mathrm{COM}}\right\rangle=\frac{3}{2} k T
$$

\section{References}

1. Vekey, K. Internal Energy Effects in Mass Spectrometry. J. Mass Spectrom. 1996, 31(5), 445-463.

2. Douglas, D. J. Applications of Collision Dynamics in Quadrupole Mass Spectrometry. J. Am. Soc. Mass Spectrom. 1998, 9(2), 101-113.

3. Drahos, L.; Vekey, K. MassKinetics. A Theoretical Model of Mass Spectra Incorporating Physical Processes, Reaction Kinetics and Mathematical Descriptions. J. Mass Spectrom. 2001, 36(3), 237-263.

4. Laskin, J.; Futrell, J. H. Collisional Activation of Peptide Ions in FT-ICR Mass Spectrometry. Mass Spectrom. Rev. 2003, 22(3), 158-181.

5. Plass, W. R.; Cooks, R. G. A Model for Energy Transfer in Inelastic Molecular Collisions Applicable at Steady State or Non-Steady State and for an Arbitrary Distribution of Collision Energies. J. Am. Soc. Mass Spectrom. 2003, 14(12), 1348 1359.

6. Gerlich, D. Inhomogeneous RF-Fields-A Versatile Tool for the Study of Processes with Slow Ions. Adv. Chem. Phys. 1992, $82,1-176$.

7. McLuckey, S. A.; Goeringer, D. E. Slow Heating Methods in Tandem Mass Spectrometry. J. Mass Spectrom. 1997, 32(5), 461-474.

8. Goeringer, D. E.; McLuckey, S. A. Kinetics of CollisionInduced Dissociation in the Paul Trap: A first-Order Model. Rapid Commun. Mass Spectrom. 1996, 10(3), 328-334.

9. Goeringer, D. E.; McLuckey, S. A. Evolution of Ion Internal Energy During Collisional Excitation in the Paul Ion Trap: A Stochastic Approach. J. Chem. Phys. 1996, 104(6), $2214-$ 2221.

10. Dodonov, A.; Kozlovsky, V.; Loboda, A.; Raznikov, V.; Sulimenkov, I.; Tolmachev, A.; Kraft, A.; Wollnik, H. A New Technique for Decomposition of Selected Ions in Molecule Ion Reactor Coupled with Ortho-Time-of-Flight 
Mass Spectrometry. Rapid Commun. Mass Spectrom. 1997, 11(15), 1649-1656.

11. Drahos, L.; Vekey, K. Special Feature: Commentary-How Closely Related are the Effective and the Real Temperature. J. Mass Spectrom. 1999, 34(2), 79-84.

12. Spangler, G. E.; Miller, R. A. Application of Mobility Theory to the Interpretation of Data Generated by Linear and RF Excited Ion Mobility Spectrometers. Int. J. Mass Spectrom. 2002, 214(1), 95-104.

13. Asano, K. G.; Butcher, D. J.; Goeringer, D. E.; McLuckey, S. A. Effective Ion Internal Temperatures Achieved via Boundary Activation in the Quadrupole Ion Trap: Protonated Leucine Enkephalin. J. Mass Spectrom. 1999, 34(6), 691-698.

14. Goeringer, D. E.; Duckworth, D. C.; McLuckey, S. A. CollisionInduced Dissociation in Quadrupole Ion Traps: Application of a Thermal Model to Diatomic Ions. J. Phys. Chem. A 2001, 105(10), 1882-1889.

15. Gabelica, V.; Karas, M.; De Pauw, E. Calibration of Ion Effective Temperatures Achieved by Resonant Activation in a Quadrupole Ion Trap. Anal. Chem. 2003, 75(19), 51525159.

16. Collette, C.; Drahos, L.; De Pauw, E.; Vekey, K. Comparison of the Internal Energy Distributions of Ions produced by Different Electrospray Sources. Rapid Commun. Mass Spectrom. 1998, 12(22), 1673-1678.

17. Collette, C.; De Pauw, E. Calibration of the Internal Energy Distribution of Ions Produced by Electrospray. Rapid Commun. Mass Spectrom. 1998, 12(4), 165-170.

18. Drahos, L.; Heeren, R. M. A ; Collette, C.; De Pauw, E.; Vekey, K. Thermal Energy Distribution Observed in Electrospray Ionization. J. Mass Spectrom. 1999, 34(12), 1373-1379.

19. Guo, X. H.; Duursma, M. C.; Kistemaker, P. G.; Nibbering, N. M. M.; Vekey, K.; Drahos, L.; Heeren, R. M. A. Manipulating Internal Energy of Protonated Biomolecules in Electrospray Ionization Fourier Transform Ion Cyclotron Resonance Mass Spectrometry. J. Mass Spectrom. 2003, 38(6), 597-606.

20. Gabelica, V.; De Pauw, E.; Karas, M. Influence of the Capillary Temperature and the Source Pressure on the Internal Energy Distribution of Electrosprayed Ions. Int. J. Mass Spectrom. 2004, 231(2/3), 189-195.

21. Raznikov, V. V.; Kozlovsky, V. I.; Dodonov, A. F.; Raznikova, M. O. Heating of Ions Moving in a Gas Under the Influence of a Uniform and Constant Electric Field. Rapid Commun. Mass Spectrom. 1999, 13(5), 370-375.

22. Tolmachev, A. V.; Chernushevich, I. V.; Dodonov, A. F.; Standing, K. G. A Collisional Focusing Ion Guide for Coupling an Atmospheric Pressure Ion Source to a Mass Spectrometer. Nucl. Instrum. Methods B 1997, 124(1), 112-119.

23. Drahos, L.; Vekey, K. Determination of the Thermal Energy and Its Distribution in Peptides. J. Am. Soc. Mass Spectrom. 1999, 10(4), 323-328.

24. Dehmelt, H. G. Radiofrequency Spectroscopy of Stored ions. I: Storage. Adv. At. Mol. Phys. 1967, 353-72.

25. Chandrasekhar, S. Stochastic Problems in Physics and Astronomy. Rev. Mod. Phys. 1943, 15, 1-83.

26. Quadrupole Mass Spectrometry and Its Applications; Dawson, P. H., Ed.; Elsevier: Amsterdam, 1976; p 349.

27. Tolmachev, A. V.; Udseth, H. R.; Smith, R. D. Charge Capacity Limitations of Radio Frequency Ion Guides in Their Use for Improved Ion Accumulation and Trapping in Mass Spectrometry. Anal. Chem. 2000, 72(5), 970-978.

28. Sannes-Lowery, K.; Griffey, R. H.; Kruppa, G. H.; Speir, J. P.; Hofstadler, S. A. Multipole Storage Assisted Dissociation, a Novel In-Source Dissociation Technique for Electrospray Ion- ization Generated Ions. Rapid Commun. Mass Spectrom. 1998, 12(23), 1957-1961.

29. Hakansson, K.; Axelsson, J.; Palmblad, M.; Hakansson, P. Mechanistic Studies of Multipole Storage Assisted Dissociation. J. Am. Soc. Mass Spectrom. 2000, 11(3), 210-217.

30. Pan, C.; Hettich R. Multipole-Storage Assisted Dissociation for Characterization of Intact Proteins. Proceedings of the 51st ASMS Conference on Mass Spectrometry and Allied Topics; Montreal, Canada, June 2003.

31. Schachterle, S. A Comparison of Excitation Methods for Collision Induced Dissociation in the Ion Trap Mass Spectrometer. Proceedings of the 41st ASMS Conference on Mass Spectrometry and Allied Topics; Chicago, IL, 1994; p 714.

32. Wells, G. J., Wang, M. U.S. Patent 5,381,006; 1995.

33. Wang, M. D.; Schachterle, S.; Wells, G. Application of Nonresonance Excitation to Ion Trap Tandem Mass Spectrometry and Selected Ejection ChemicalI. J. Am. Soc. Mass Spectrom. 1996, 7(7), 668-676.

34. Plass, W. R. Theory of Dipolar dc Excitation and dc Tomography in the rf Quadrupole Ion Trap. Int. J. Mass Spectrom. 2000, 202(1/3), 175-197.

35. Schnier, P. D.; Price, W. D.; Jockusch, R. A.; Williams, E. R. Blackbody Infrared Radiative Dissociation of Bradykinin and Its Analogues: Energetics, Dynamics, and Evidence for SaltBridge Structures in the Gas Phase. J. Am. Chem. Soc. 1996, 118(30), 7178-7189.

36. Price, W. D.; Schnier, P. D.; Jockusch, R. A.; Strittmatter, E. F.; Williams, E. R. Unimolecular Reaction Kinetics in the HighPressure Limit Without Collisions. J. Am. Chem. Soc. 1996, 118(43), 10640-10644.

37. Gross, D. S.; Zhao, Y. X.; Williams, E. R. Dissociation of Heme-Globin Complexes by Blackbody Infrared Radiative Dissociation: Molecular Specificity in the Gas Phase? J. Am. Soc. Mass Spectrom. 1997, 8(5), 519-524.

38. Dunbar, R. C.; McMahon, T. B. Activation of Unimolecular Reactions by Ambient Blackbody Radiation. Science 1998, 279(5348), 194-197.

39. Stevens, S. M.; Dunbar, R. C.; Price, W. D.; Sena, M.; Watson, C. H.; Nichols, L. S.; Riveros, J. M.; Richardson, D. E.; Eyler, J. R. Blackbody Infrared Radiative Dissociation of Partially Solvated tris(2,2'-Bipyridine)Ruthenium(II) Complex Ions. J. Phys. Chem. A 2002, 106(42), 9686-9694.

40. Dunbar, R. C. BIRD (Blackbody Infrared Radiative Dissociation): Evolution, Principles, and Applications. Mass Spectrom. Rev. 2004, 23(2), 127-158.

41. Vilkov, A. N.; Păśa-Tolic, L.; Bogdanov, B.; Ahn, S.; Prior, D. C.; Anderson, G. A.; Masselon, C. D.; Smith, R. D. Multistage External Preselection of Ions for Increased Sensitivity of LC-FTICR MS/MS. Proceedings of the 51st ASMS Conference on Mass Spectrometry and Allied Topics; Montreal, Canada, June 2003.

42. Vilkov, A. N.; Bogdanov, B.; Pasa-Tolic, L., Ahn, S.; Prior, D. C.; Anderson, G. A.; Masselon, C. D.; Smith, R. D. Multistage External Preselection of Ions for Increased Sensitivity of LC-FTICR MS/MS, unpublished.

43. Covey, T.; Douglas, D. J. Collision Cross-Sections for Protein Ions. J. Am. Soc. Mass Spectrom. 1993, 4(8), 616-623.

44. Chernushevich, I. V.; Thomson, B. A. Collisional Cooling of Large Ions in Electrospray Mass Spectrometry. Anal. Chem. 2004, 761754-1760.

45. Price, W. D.; Schnier, P. D.; Williams, E. R. Binding Energies of the Proton-Bound Amino Acid Dimers Gly-Gly, Ala-Ala, Gly-Ala, and Lys-Lys Measured by Blackbody Infrared Radiative Dissociation. J. Phys. Chem. B 1997, 101(4), 664-673.

46. Price, W. D.; Williams, E. R. Activation of Peptide Ions by Blackbody Radiation: Factors that Lead to Dissociation Kinet- 
ics in the Rapid Energy Exchange Limit. J. Phys. Chem. A 1997, 101(47), 8844-8852.

47. Schnier, P. D.; Price, W. D.; Strittmatter, E. F.; Williams, E. R. Dissociation Energetics and Mechanisms of Leucine Enkephalin $(\mathrm{M}+\mathrm{H})^{+}$and $(2 \mathrm{M}+\mathrm{X})^{+}$ions $(\mathrm{X}=\mathrm{H}, \mathrm{Li}, \mathrm{Na}, \mathrm{K}$, and $\mathrm{Rb})$ Measured by Blackbody Infrared Radiative Dissociation. J. Am. Soc. Mass Spectrom. 1997, 8(8), 771-780.

48. Paradisi, C.; Todd, J. F. J.; Traldi, P.; Vettori, U. Boundary Effects and Collisional Activation in a Quadrupole Ion Trap. Org. Mass Spectrom. 1992, 27(3), 251-254.
49. Paradisi, C.; Todd, J. F. J.; Vettori, U. Comparison of Collisional Activation by the Boundary Effect Versus Tickle Excitation in an Ion Trap Mass-Spectrometer. Org. Mass Spectrom. 1992, 27(11), 1210-1215.

50. Creaser, C. S.; Oneill, K. E. Boundary-Effect Activated Dissociation in Ion-Trap Tandem Mass-Spectrometry. Org. Mass Spectrom. 1993, 28(5), 564-569.

51. Vachet, R. W.; Glish, G. L. Boundary-Activated Dissociation of Peptide Ions in a Quadrupole Ion Trap. Anal. Chem. 1998, $70(2), 340-346$. 